\title{
MODELAGEM DINÂMICA DA SECAGEM DE PASTA EM LEITO DE JORRO
}

\author{
C.A.M. SILVA*, F.B. FREIRE, J.T. FREIRE \\ Universidade Federal de São Carlos, Departamento de Engenharia Química \\ *e-mail: carlosmoreisilva@gmail.com
}

\begin{abstract}
RESUMO
Apesar da literatura reportar vários modelos matemáticos empregados para a secagem de pasta em leito de jorro, poucos trabalhos propõem estudar o regime transiente envolvendo mudanças na dinâmica de secagem. Portanto, neste trabalho foi realizado um estudo experimental e de simulação sobre o processo de secagem de pasta em leito de jorro com partículas inertes, onde os testes foram conduzidos de modo a provocar mudanças na dinâmica de secagem para se procurar entender o que ocorre no processo durante períodos em que o equipamento passa por estados transientes. Para isto, foram realizados experimentos de secagem com perturbação na vazão de alimentação da pasta. Os ensaios foram realizados com temperaturas do gás de entrada de $70^{\circ} \mathrm{C}$ e $100^{\circ} \mathrm{C}$, e velocidades do gás de entrada de $20 \%$ e $30 \%$ acima da mínima de jorro. A pasta utilizada foi uma suspensão de carbonato de cálcio com uma concentração percentual de sólidos de 5\% $\mathrm{m} / \mathrm{m}$, e esferas de vidro como partículas inertes. Para descrever a dinâmica de transferência de calor e massa no leito, foi utilizado um modelo semi-empírico que consiste de um sistema de equações diferenciais ordinárias acoplado com uma equação empírica para determinar a taxa de evaporação de água. Para a solução do sistema, foi construído um código computacional em linguagem de programação FORTRAN 90 utilizando-se a subrotina DIVIPAG da biblioteca do IMSL. Foram realizadas análises comparativas entre os dados experimentais e simulados, e verificou-se que o modelo adaptado representa bem o processo de secagem em diferentes condições experimentais.
\end{abstract}

\section{INTRODUÇÃO}

Segundo Medeiros et al. (2001), desde o seu surgimento por volta de 1960, quando pesquisadores da antiga URSS do Instituto de Tecnologia de Leningrado, usaram para a secagem de lacas e tintas, a técnica de secagem de pastas em leitos de jorro com materiais inertes vem sendo amplamente estudada no Brasil e no mundo. Devido a essa técnica provocar uma operação cíclica das partículas, favorecendo um alto grau de mistura e resultando em elevadas taxas de transferência de calor e massa, baixo custo de instalação e facilidade de operação, o equipamento leito de jorro tem sido fortemente utilizado, tanto no meio acadêmico como industrial, para a secagem de diversos tipos de materiais pastosos. Dentre eles destacam-se: os produtos químicos inorgânicos (suspensões ou soluções de $\mathrm{Al}(\mathrm{OH})_{3}, \mathrm{CaCO}_{3}, \mathrm{Al}_{2} \mathrm{O}_{3}, \mathrm{NaCl}$, etc); pastas alimentícias (polpas de frutas tropicas, leites, ovo homogeneizado, etc); produtos farmacêuticos (extratos vegetais, licor preto, etc) e outros subprodutos (sangue animal, lodo de esgoto, efluente industrial de amido, etc). (FREIRE et al., 2012). 
Entretanto, apesar do desenvolvimento de diversos estudos experimentais a cerda do comportamento fluidodinâmico do leito na presença de pastas, psicrometria, qualidade do pó, composição da pasta, eficiência energética, etc, poucas tentativas sobre a modelagem dinâmica do processo têm aparecido na literatura (FERNANDES, 2005; DANTAS, 2013; FILHO, 2013; VIEIRA, 2015). De fato, a formulação de modelos matemáticos para descrever adequadamente $\mathrm{o}$ processo de secagem de pastas em leito de jorro com partículas inertes é bastante complexa. Devido aos fenômenos simultâneos de transporte de momento, calor e massa, e aos fenômenos de interações puramente físico-químicas causadas pelas forças de adesão do filme formado nas partículas inertes, e também a forças de cisalhamento que atuam no filme líquido, o processo torna-se ainda altamente não-linear. Portanto, ainda existe uma grande necessidade de obtenção de bons modelos matemáticos baseados nas leis de conservação para simular, monitorar e controlar o processo de secagem de pastas em leitos de jorro.

Para poder monitorar e controlar a variável mais importante do processo de secagem de pasta, ou seja, o conteúdo de umidade do pó, é de fundamental importância ter um modelo matemático que descreva fisicamente e consistentemente, os fenômenos simultâneos envolvidos no processo quando ocorreram perturbações em algumas variáveis que são susceptíveis de se alterarem ao longo do tempo, por exemplo, a vazão de alimentação de pasta, a temperatura e umidade absoluta do gás na entrada, etc.

Portanto, visando obter um melhor entendimento sobre a modelagem matemática para o monitoramento e controle do processo de secagem de pasta em leito de jorro, o objetivo do presente trabalho foi realizar um estudo experimental e de simulação provocando mudanças na dinâmica de secagem por meio de perturbação na vazão de alimentação de pasta, com intuito de compreender o que ocorre no processo quando este passa por períodos transientes. Utilizou-se o modelo de Freire et al. (2009), modificandose alguns termos e parâmetros do modelo.

\section{MATERIAIS E MÉTODOS}

\subsection{Unidade Experimental}

A unidade experimental utilizada para a realização dos ensaios encontra-se no Centro de Secagem de Pastas e Sementes do DEQ/UFSCar, conforme mostrado na Figura 1:

Figura 1 - Representação da unidade experimental.

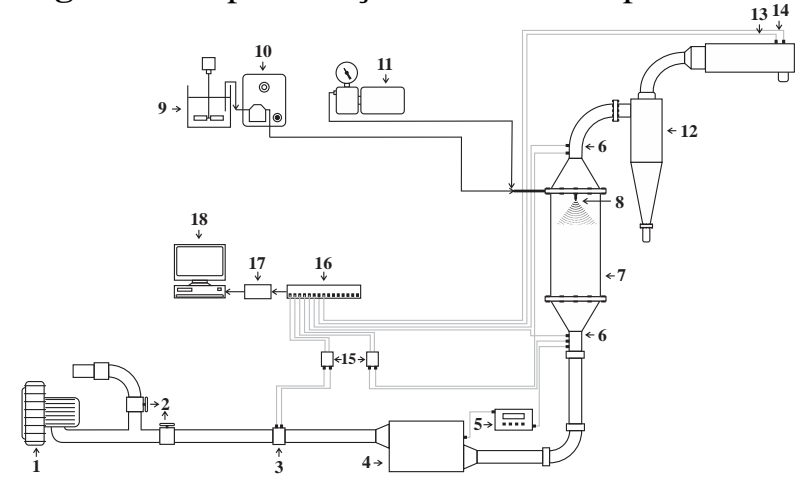

(1) soprador radial, (2) válvulas do tipo gaveta, (3) placa de orifício, (4) trocador de calor, (5) controlador de temperatura, (6) termopares, (7) leito de jorro, (8) bico atomizador de pasta, (9) reservatório de pasta, (10) bomba peristáltica, (11) compressor de ar, (12) ciclone, (13) termopar de bulbo seco, (14) termopar de bulbo úmido, (15) transdutores de pressão, (16) condicionador de sinais, (17) placa de aquisição de dados, (18) microcomputador.

O equipamento utilizado nos ensaios de secagem foi um leito de jorro de geometria cônico-cilíndrica (7) construído com peças em aço inox e unidas por flanges. $\mathrm{O}$ leito de jorro possui a base cônica com altura de $12,0 \mathrm{~cm}$, ângulo incluso de $60^{\circ}$, diâmetro de entrada de $3,0 \mathrm{~cm}$, coluna cilíndrica de $50,0 \mathrm{~cm}$, cone superior de $13,0 \mathrm{~cm}$ de altura com ângulo incluso de $60^{\circ}$ e diâmetro de saída de $5,0 \mathrm{~cm}$. 


\subsection{Materiais}

A pasta utilizada nos experimentos de secagem foi uma suspensão de Carbonato de Cálcio com uma concentração percentual em massa de $5 \% \mathrm{~m} / \mathrm{m}$. O critério de escolha desta pasta foi devido ao fato de que a mesma (do ponto de vista da secagem) não apresenta grandes problemas operacionais e de instabilidade, e a facilidade da preparação da suspensão com uma concentração prédefinida. Como partículas inertes utilizaram-se $1,5 \mathrm{~kg}$ de esferas de vidro com $d_{p}$ médio de 2,2 $\mathrm{mm}$ e $\rho_{p}$ de $2512 \mathrm{~kg} / \mathrm{m}^{3}$.

\subsection{Sistema de Aquisição de Dados}

A unidade experimental está instrumentada de forma a obterem-se dados de pressão e temperatura em diferentes pontos conforme mostrado na Figura 1. Os dados de pressão foram obtidos através de dois transdutores de pressão (um para a medida da queda de pressão no leito e outro para a medida da vazão de ar na placa de orifício). Para as medidas de temperatura foram utilizados cinco termopares do tipo $\mathrm{T}$, sendo dois deles localizados na entrada e saída do leito, dois para medidas da temperatura de bulbo seco e bulbo úmido na saída do ciclone e o outro para uso do controlador de temperatura. Os termopares de bulbo seco e úmido na saída do ciclone foram utilizados para o cálculo de propriedades psicrométricas do ar.

Todos os sensores de pressão e de temperatura estão conectados a um modulo condicionador de sinais e uma placa de aquisição de dados inserida no microcomputador. Para a aquisição em tempo real das variáveis mencionadas anteriormente, foram elaborados instrumentos virtuais no software Labview 6.1, onde foi possível adquirir e armazenar automaticamente de 10 em 10 segundos e com uma taxa de amostragem de $400 \mathrm{~Hz}$, os valores de temperatura e pressão ao longo do processo.

\subsection{Caracterização da Unidade Experimental}

A caracterização da unidade experimental consistiu na obtenção das curvas fluidodinâmicas do leito de jorro para as condições de temperaturas e partículas inertes que foram empregadas nos experimentos de secagem. Estas curvas foram obtidas de acordo com a metodologia proposta por Mathur e Epstein (1974).

\subsection{Procedimento Experimental de Secagem}

As condições experimentais utilizadas nos ensaios de secagem são mostradas na Tabela 1:

Tabela 1 - Condições experimentais de secagem.

\begin{tabular}{ccc}
\hline $\mathrm{Tg}_{\mathrm{e}}\left({ }^{\circ} \mathrm{C}\right)$ & $\mathrm{u}(\mathrm{m} / \mathrm{s})$ & $\mathrm{Q}_{\text {susp }}(\mathrm{mL} / \mathrm{min})$ \\
\hline \multirow{2}{*}{70} & \multirow{2}{*}{$1,2 \mathrm{u}_{\mathrm{mj}}$} & $5 \rightarrow 15$ \\
\cline { 2 - 2 } & \multirow{2}{*}{100} & $15 \rightarrow 5$ \\
\cline { 2 - 2 } & \multirow{2}{*}{$1,3 \mathrm{u}_{\mathrm{mj}}$} & $5 \rightarrow 15$ \\
\cline { 3 - 3 } & & $20 \rightarrow 5$ \\
\hline
\end{tabular}

Os experimentos foram realizados com duas condições de temperatura e velocidade do gás na entrada do leito. Após colocado o equipamento em funcionamento, esperava-se o sistema entrar em regime permanente. Atingido este estado, iniciava-se a alimentação da pasta. Esperava-se o sistema atingir o regime permanente, e logo atingido esse estado ou quando o leito começava a apresentar problemas operacionais e de instabilidades, mudava-se a vazão de alimentação da pasta. $\mathrm{O}$ processo ocorria até atingir um novo regime permanente ou quando o leito apresentasse novos problemas operacionais e de instabilidades. A alimentação da pasta no leito ocorreu de forma contínua durante todo o período do processo. Sempre antes de cada experimento, realizava-se a determinação da vazão de alimentação da pasta com teste em proveta e cronômetro. Durante os ensaios, foram monitoradas e coletadas ao longo do tempo, as principais variáveis do processo como por exemplo: $\mathrm{Tg}_{\mathrm{e}}, \mathrm{Tg}_{\mathrm{s}}, \mathrm{UA}_{\mathrm{s}}, \mathrm{UR}_{\mathrm{s}}, \mathrm{Wg}_{\mathrm{e}}$, 
$\Delta \mathrm{P}_{\mathrm{L}}$ e Xbu. Para a determinação do conteúdo de umidade do pó em base úmida, amostras foram coletadas na saída do ciclone de 5 em 5 minutos. O conteúdo de umidade do pó foi determinado em estufa a $105^{\circ} \mathrm{C} / 24 \mathrm{~h}$.

\subsection{Modelagem do Problema}

Para descrever a dinâmica de transferência de calor e massa no leito de jorro, utilizou-se um modelo CST transiente adaptado e baseado nas leis da conservação de massa e energia proposto por Freire et al. (2009). Na Figura 2, é mostrada uma representação esquemática do sistema a ser modelado.

Figura 2 - Representação esquemática do leito de jorro.

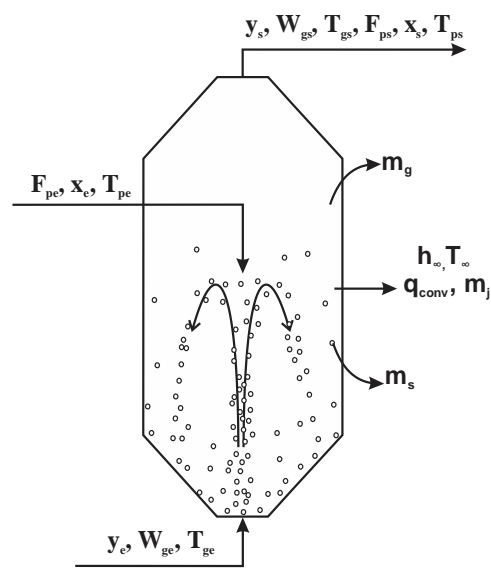

As principais considerações realizadas para a construção do modelo CST são:

1. o leito de jorro comporta-se como um tanque perfeitamente agitado, ou seja, não existem variações espaciais de umidade do gás e do pó, e de temperatura no interior do leito.

2. a mistura gasosa comparta-se como gás ideal.

3. existe acúmulo de pasta no interior do leito de jorro.

4. a temperatura de saída do pó é igual a temperatura de saída do gás.

Realizando-se um balanço global de energia, e um balanço de massa de água no leito de jorro para as fases gasosa e líquida, e a partir das considerações realizadas, tem-se o seguinte conjunto de equações diferenciais ordinárias e suas condições iniciais:

$$
\begin{aligned}
& \frac{d y_{s}}{d t}=\frac{1}{m_{g}}\left\{W_{g e} y_{e}-W_{g s} y_{s}+k^{y_{s}, T_{g s}}\right\} \\
& \frac{d x_{s}}{d t}=\frac{1}{m_{p}}\left\{F_{p e}\left(x_{e}-x_{s}\right)-k^{x_{s}}\right\} \\
& \frac{d T_{g s}}{d t}=\frac{W_{g e} C p_{g e} T_{g e}+F_{p e} C p_{p e} T_{p e}-W_{g s} C p_{g s} T_{g s}}{m_{g} C p_{g s}+m_{s} C p_{s}+m_{j} C p_{j}} \\
& +\frac{-F_{p s} C p_{p s} T_{g s}-k^{y_{s}, T_{g s}} \Delta H^{v}-q_{c o n v}}{m_{g} C p_{g s}+m_{s} C p_{s}+m_{j} C p_{j}} \\
& y_{s}(\mathrm{t}=0)=\mathrm{y}_{\mathrm{s} 0} \\
& x_{s}(\mathrm{t}=0)=x_{e} \\
& T_{g s}(\mathrm{t}=0)=T_{\mathrm{gs} 0}
\end{aligned}
$$

Nas Equações 1.a até 1.c, a letra " $k$ " corresponde a taxa de evaporação de água. Como ainda não se conhece uma equação para descrever a taxa de evaporação de água em função das condições operacionais, utilizou-se equações empíricas de forma exponencial, conforme mostradas abaixo:

$0 \leq t \leq t_{p}$

$k^{y_{s}, T_{g s}}=k_{1}^{y_{s}, T_{g s}}=a_{1}\left(1-\mathrm{e}^{-b_{1} t}\right)$

$k^{x_{s}}=k_{1}^{x_{s}}=k_{1}^{y_{s}, T_{g s}}$

$t>t_{p}$

$k^{y_{s}, T_{g s}}=k_{2}^{y_{s}, T_{g s}}=k_{1}^{y_{s}, T_{g s}}\left(t=t_{p}\right) \pm a_{2}\left(1-\mathrm{e}^{-b_{2}\left(t-t_{p}\right)}\right)$

$k^{x_{s}}=k_{2}^{x_{s}}=k_{1}^{y_{s}, T_{g s}}\left(t=t_{p}\right) \pm k_{2}^{y_{s}, T_{g s}}\left(t=t_{p}\right)$

onde o subscrito "1" e "2", referem-se a primeira e segunda condição de vazão de alimentação de pasta, respectivamente.

Para o período de $0 \leq \mathrm{t} \leq \mathrm{t}_{\mathrm{p}}$, considerouse que não existe acumulo de pasta no leito, e o conteúdo de umidade do pó foi descrita pela equação algébrica proposta por Almeida (2009). Para o período de $t>t_{p}$, considerou-se 
que existe acumulo de pó e o conteúdo de umidade do pó foi descrito pela Equação 1.b. Os sinais de \pm nas Equações 2.c e 2.d, referemse ao degrau de perturbação na vazão de alimentação da pasta. Se "+”, ocorreu um aumento na vazão de alimentação da pasta e se “_" significa que ocorreu uma diminuição na vazão de alimentação da pasta. Para resolver o modelo, foi construído um algoritmo em linguagem de programação Fortran 90, utilizando-se a subrotina DIVPAG da biblioteca do IMSL. As equações auxiliares que compõem o modelo CST, não foram mostradas aqui, mas podem ser encontradas no trabalho de Almeida (2009).

\section{RESULTADOS E DISCUSSÃO}

\subsection{Caracterização Fluidodinâmica}

A curva fluidodinâmica obtida (não mostrada) apresentou comportamento característico de uma curva de leito de jorro de acordo com Mathur e Epstein (1974). Na Tabela 2, são mostrados alguns parâmetros obtidos a partir da curva fluidodinâmica. Observa-se que para as duas temperaturas, não obtiveram-se diferenças significativas na velocidade mínima de jorro, e portanto, a temperatura do gás de entrada praticamente não afeta este parâmetro.

Tabela 2 - Parâmetros fluidodinâmicos obtidos do leito de jorro.

\begin{tabular}{cccc}
\hline $\mathrm{T}_{\mathrm{ge}}\left({ }^{\circ} \mathrm{C}\right)$ & $\mathrm{P}_{\max }(\mathrm{Pa})$ & $\mathrm{P}_{\mathrm{mj}}(\mathrm{Pa})$ & $\mathrm{u}_{\mathrm{mj}}(\mathrm{m} / \mathrm{s})$ \\
\hline 70 & 2093,24 & 724,39 & 17,00 \\
\hline 100 & 1805,32 & 820,60 & 16,40 \\
\hline
\end{tabular}

\subsection{Análise da Simulação da Secagem}

Para a realização da simulação do problema, os valores dos coeficientes de ajuste das Equações 2.a-2.d, bem como os coeficientes de transferência de calor paredeleito foram ajustados de forma que o modelo CST pudesse representar satisfatoriamente os dados experimentais.
Nas condições de $70^{\circ} \mathrm{C}\left(\mathrm{u}=1,2 \mathrm{u}_{\mathrm{mj}}\right)$ e $100^{\circ} \mathrm{C}\left(\mathrm{u}=1,3 \mathrm{u}_{\mathrm{mj}}\right)$, as vazões máximas de pasta que podem ser alimentadas no leito para os experimentos com mudanças na dinâmica de secagem são de $15 \mathrm{~mL} / \mathrm{min}$ e $20 \mathrm{~mL} / \mathrm{min}$, respectivamente. Portanto, os experimentos foram conduzidos próximo da capacidade máxima de secagem do leito.

Nas Figuras 3 a 6 (itens "a", "b”), são mostradas as comparações entre os dados experimentais e simulados para a umidade absoluta do gás na saída do leito, do conteúdo de umidade do pó em base úmida, da temperatura do gás e da umidade relativa do gás na saída do leito. É observado que para baixas vazões de alimentação da pasta $(5 \mathrm{~mL} / \mathrm{min})$, existem poucos desvios entre os dados experimentais e simulados. A medida que aumenta-se a vazão de alimentação da pasta ( 15 ou $20 \mathrm{~mL} / \mathrm{min})$, desvios significativos começam a surgir. Como o leito está operando próximo da sua capacidade máxima, o sistema começa a apresentar problemas operacionais e de instabilidade, ou seja, os parâmetros fluidodinâmicos começam a mudar significativamente ao longo do tempo. Essas alterações na fluidodinâmica do leito não são incorporadas ao modelo, e pode estar provocando os desvios consideráveis entres os dados experimentais e simulados.

Nas Figuras 3 a 6 (itens “c") são mostradas as taxas de evaporação de água calculadas a partir das Equações 2.a-2.d. É observado que a taxa de evaporação de água apresenta um perfil semelhante ao perfil de umidade absoluta do gás na saída do leito.

O comportamento da queda de pressão do leito e da vazão do gás em função do tempo são mostradas no item "d" das Figuras 4 a 7. Pode-se ver que em todos os experimentos ocorrem mudanças nos parâmetros fluidodinâmicos o leito de jorro.

Para os experimentos com degrau positivo na vazão de alimentação da pasta, conforme pode-se ver nas Figuras 3.a e 5.a, a umidade absoluta do gás na saída do leito 
aumenta a partir da umidade absoluta do gás na entrada $e$ atinge rapidamente $o$ regime permanente. Devido à alta vazão mássica do gás de escoamento e o fato das partículas inertes no início do experimento estarem bem quentes, ocorre rápida transferência de massa de água da fase líquida para a fase vapor. Em razão das altas taxas de transferência de calor e massa que se obtém no leito de jorro, a fração mássica de água liquida inicial da pasta diminui bruscamente $\left(\mathrm{Xbu}_{0}=95 \%\right)$ para um valor que permanece praticamente constante para o primeiro período de secagem $(\mathrm{Xbu}=0,2$ $0,3 \%)$.

Conforme pode-se ver nas Figuras 3.b e 5.b, a umidade relativa do gás na saída do leito aumenta de forma mais lenta em relação a umidade absoluta, devido a lenta diminuição da temperatura do gás na saído do leito até atingir praticamente o regime permanente. A medida em que vai ocorrendo a injeção de pasta no sistema, o leito de jorro vai ficando mais frio, e a pressão de vapor de água aumenta até atingir o regime permanente.

De acordo com as Figuras 3.d e 5.d, percebe-se ainda que não ocorrem mudanças significativas no comportamento dos parâmetros fluidodinâmicos. Como o ar de secagem ainda apresenta um potencial para continuar o processo de secagem, aumentou-se a vazão de alimentação de pasta.

Conforme pode-se ver nas Figuras 3.a e 5.a, este degrau positivo provoca um rápido aumento da umidade absoluta do gás na saída até permanecer constante. Este rápido aumento, deve-se também a alta vazão do gás e pelo fato de que o ar ainda apresentar um forte potencial para absorver umidade, ocorrendo uma rápida transferência de massa de água da fase líquida para a fase vapor.

A temperatura do gás na saída do leito diminui mais ainda devido ao aumento da vazão de alimentação da pasta, que provoca um abaixamento das temperaturas das partículas e das paredes do leito. A umidade relativa na saída do leito aumenta lentamente devido à elevação da pressão de vapor de água no leito, de acordo com as Figuras 3.b e 5.b.

Figura 3 - Resultados a $70^{\circ} \mathrm{C}$ e $\mathrm{Qs}_{\mathrm{usp}}=5 \mathrm{~mL} / \mathrm{min}$ para $\mathrm{Q}_{\text {susp }}=15 \mathrm{~mL} / \mathrm{min}$.
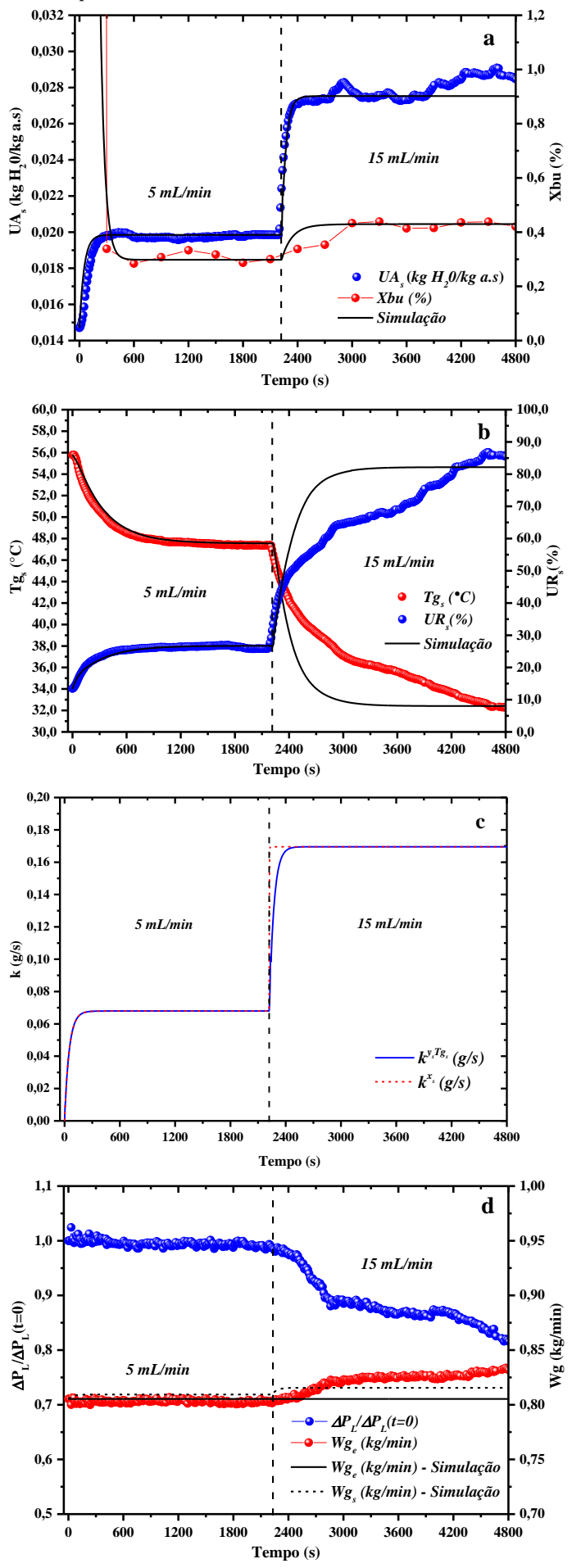
Figura 4 - Resultados a $70^{\circ} \mathrm{C}$ e $Q_{\text {susp }}=15 \mathrm{~mL} / \mathrm{min}$ para $\mathrm{Q}_{\text {susp }}=5 \mathrm{~mL} / \mathrm{min}$.
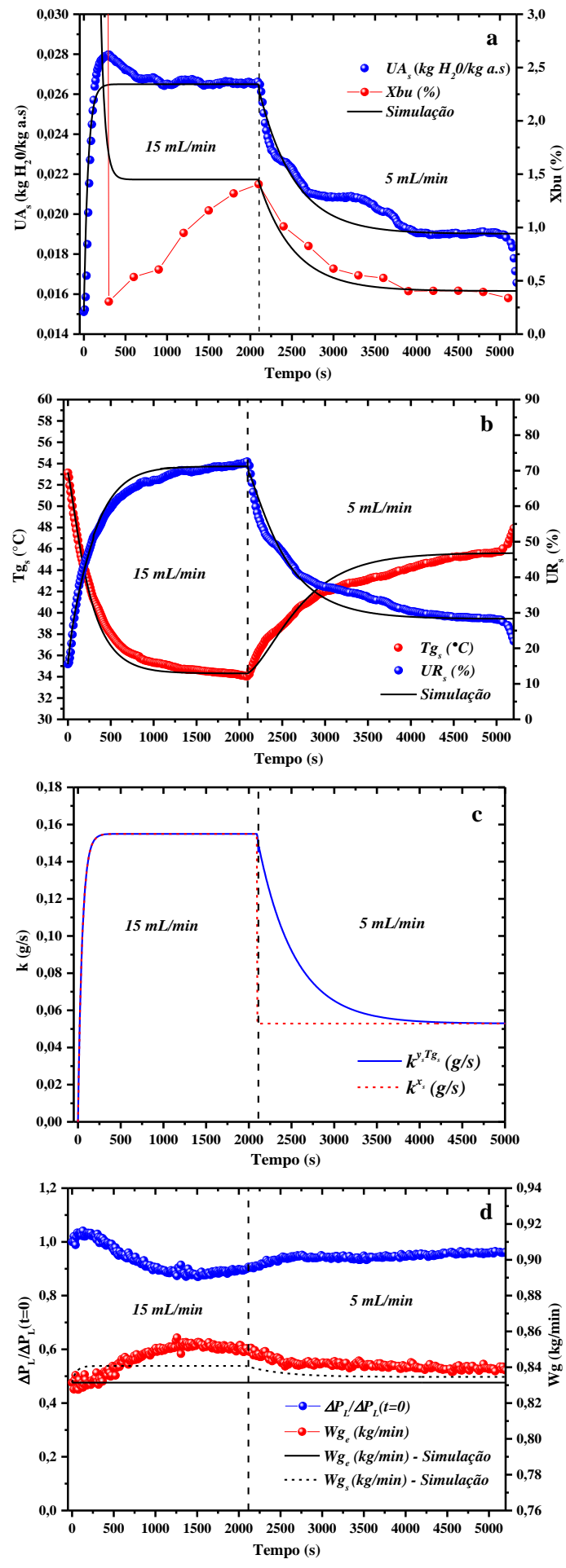

Como o ar apresenta uma capacidade de secagem reduzida, o regime permanente tornase mais demorado para ser atingido. $\mathrm{O}$ aumento da vazão de alimentação de pasta ainda foi suficiente para provocar mudanças significativas dos parâmetros fluidodinâmicos, conduzindo o sistema ao colapso do jorro para tempos suficientemente grandes, conforme mostrado nas Figuras 3.d e 5.d.

Para os experimentos com degrau negativo na vazão de alimentação da pasta, e conforme mostrada nas Figuras 4.a e 6.a, a umidade absolta do gás na saída do leito aumenta a partir da umidade absoluta do gás na entrada e rapidamente atinge o regime permanente. O conteúdo de umidade do pó na saída do ciclone tende a aumentar enquanto ocorrer a injeção de alta vazão de alimentação de pasta no leito. Esse aumente do conteúdo de umidade do pó experimental não é previsto pelo modelo CST. Para essas condições, acredita-se que está ocorrendo um processo de secagem e umedecimento do pó. Os fenômenos de umedecimento não são incorporados no modelo CST, causando grandes desvios entres os dados experimentais e simulados. Embora esteja operando em condições próximas de instabilidades, o leito de partículas não entrou em colapso. A temperatura do gás na saída do leito diminui ao longo do tempo até atingir o regime permanente, enquanto que a umidade relativa do gás na saída do leito aumenta, tendendo ao regime permanente para tempos suficientemente grandes de acordo com as Figuras 4.b e 6.b.

Como o equipamento opera próximo da região do surgimento de problemas operacionais e de instabilidades, conforme mostrado nas Figuras 4.d e 6.d, surgem desvios significativos entre os dados experimentais e simulados, sendo mais evidenciado para a temperatura de $100^{\circ} \mathrm{C}$ 
Figura 5 - Resultados a $100^{\circ} \mathrm{C}$ e $\mathrm{Q}_{\text {susp }}=5 \mathrm{~mL} / \mathrm{min}$ para $\mathrm{Q}_{\text {susp }}=15 \mathrm{~mL} / \mathrm{min}$.
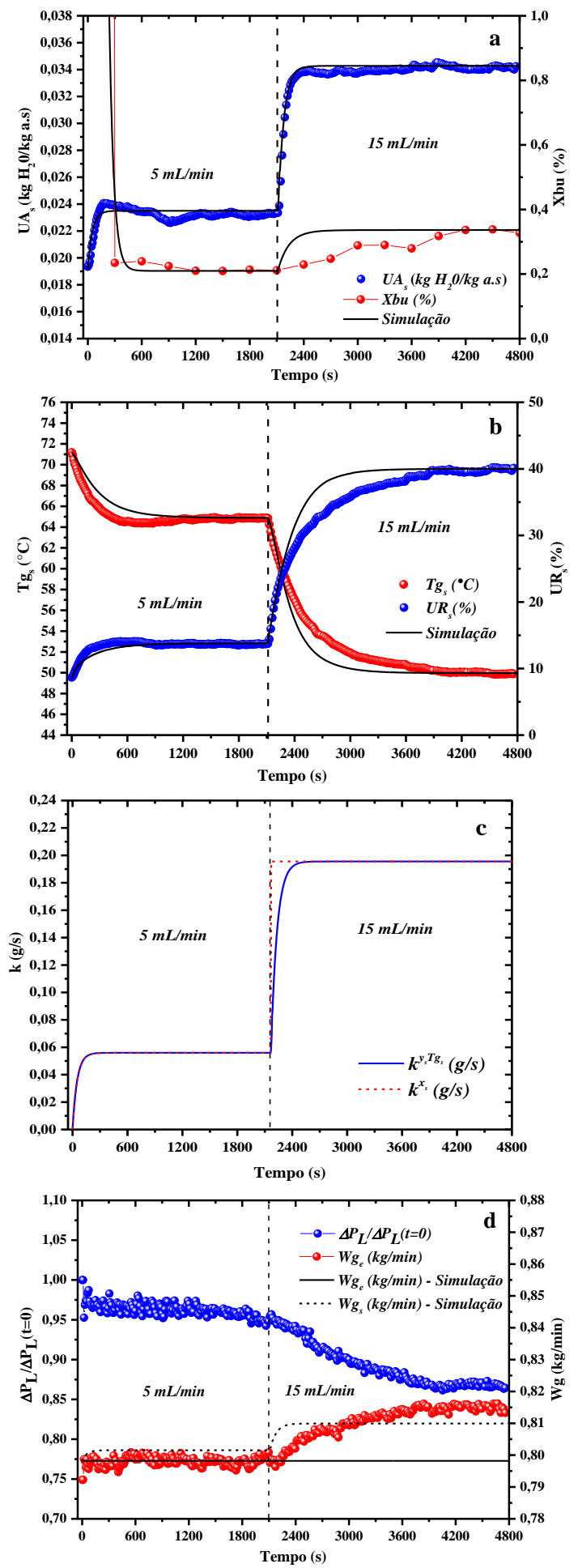

Figura 6 - Resultados da simulação a $100^{\circ} \mathrm{C}$ e $\mathrm{Q}_{\text {susp }}=20 \mathrm{~mL} / \mathrm{min}$ para $\mathrm{Q}_{\text {susp }}=5 \mathrm{~mL} / \mathrm{min}$.
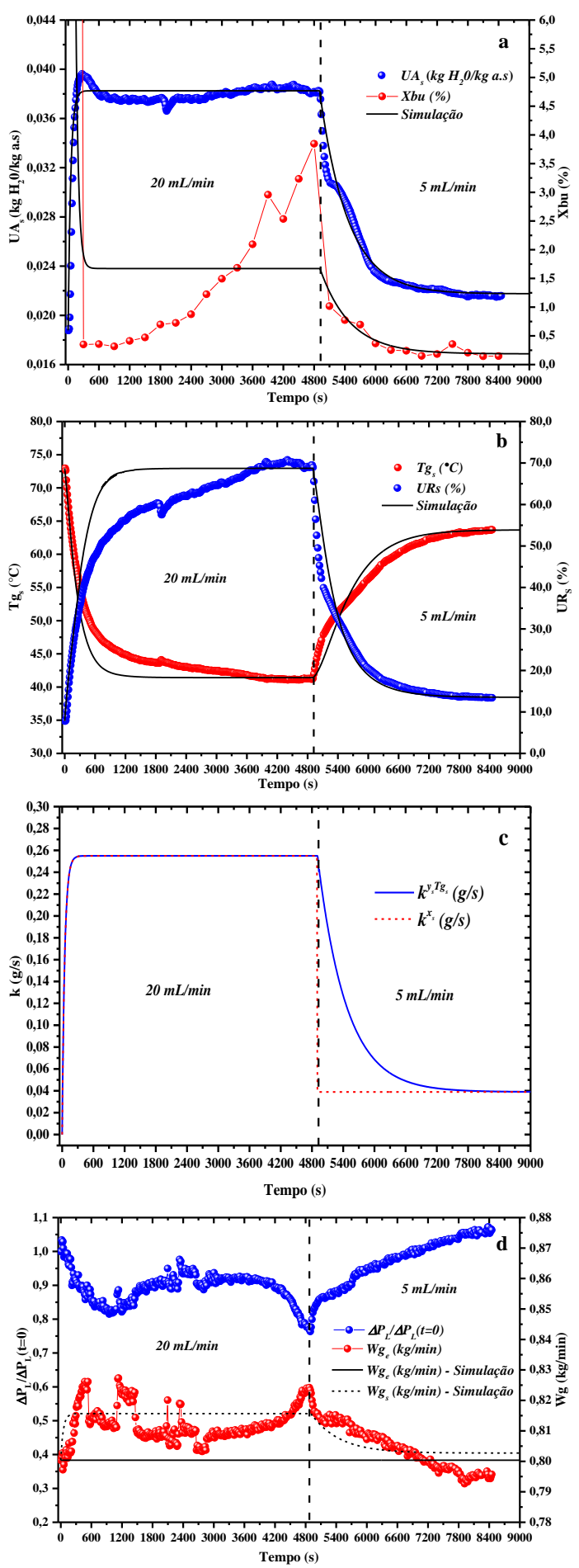

$\mathrm{Na}$ Tabela 3 são apresentados os coeficientes de determinação calculados a partir dos dados experimentais e simulados. 
Conforme pode-se ver, para todas as condições experimentais de secagem, o modelo CST consegue representar satisfatoriamente os dados experimentais de umidade absoluta, umidade relativa e a temperaturas do gás na saída do leito, com $\mathrm{R}^{2}$ superior a 0,9 . Porém, o modelo CST não consegue prever de forma satisfatória os dados experimentais do conteúdo de umidade do pó em base úmida, apresentando coeficientes de determinação inferiores a 0,5 .

Tabela 3 - Coeficientes de determinação.

\begin{tabular}{c|c|c|c|c|c}
\hline \multirow{2}{*}{ Tge } & \multirow{2}{*}{$\mathrm{Q}_{\text {susp }}$} & \multicolumn{4}{|c}{$\mathrm{R}^{2}$} \\
\cline { 3 - 6 } & & UAs & URs & Tgs & Xbu \\
\hline \multirow{2}{*}{70} & $5 \rightarrow 15$ & 0,9905 & 0,9374 & 0,9564 & 0,4796 \\
\cline { 2 - 6 } & $15 \rightarrow 5$ & 0,9808 & 0,9915 & 0,9804 & 0,2917 \\
\hline \multirow{2}{*}{100} & $5 \rightarrow 15$ & 0,9972 & 0,9878 & 0,9885 & 0,3466 \\
\cline { 2 - 6 } & $20 \rightarrow 5$ & 0,9923 & 0,9627 & 0,9685 & 0,2904 \\
\hline
\end{tabular}

De fato, modificações precisam ser realizadas na Equação 1.b e nas equações auxiliares do modelo CST, bem com propor uma equação que descreva a taxa de evaporação de água em função das condições operacionais do processo de secagem de pasta, de modo a se obter uma melhor correlação entre os dados experimentais e simulados.

\section{CONCLUSÃO}

Como já reportado em muitos trabalhos na literatura, a presença da pasta no sistema, dependendo da vazão de alimentação de pasta, altera significativamente o comportamento fluidodinâmico do leito de jorro. Essa dependência fluidodinâmica não é incorporada no modelo, o que faz com que o mesmo não consiga representar adequadamente o processo de secagem de pasta para condições onde o leito opera próximo das situações de instabilidades. Foi possível observar mudanças no conteúdo de umidade do pó, devido a alterações na dinâmica de secagem provocadas por perturbações na vazão de alimentação da pasta. O modelo CST foi capaz de prever de forma coerente e está fisicamente consistente com dados experimentais de umidade absoluta, umidade relativa e a temperaturas do gás na saída do leito. Com relação ao conteúdo de umidade do pó, grandes desvios foram observados entre os dados experimentais e simulados. Informações sobre o comportamento fluidodinâmico do leito e também sobre o umedecimento do pó devem ser incorporadas no modelo, e melhorar a equação que descreve a taxa de evaporação de água, de forma a predizer satisfatoriamente todas as principais variáveis envolvidas no processo de secagem de pasta em leito de jorro.

\section{NOMENCLATURA}

a Coeficiente de ajuste (g/s)

b Coeficiente de ajuste (1/s)

$d_{p} \quad$ Diâmetro médio das partículas $(\mathrm{mm})$

$\mathrm{Fp}_{\mathrm{e}} \quad$ Vazão mássica da pasta na entrada $(\mathrm{g} / \mathrm{s})$

$\mathrm{h}_{\infty} \quad$ Coeficiente transferência de calor por convecção $\left(\mathrm{J} / \mathrm{scm}^{2} \mathrm{~K}\right)$

$\mathrm{k}^{\mathrm{xs}} \quad$ Taxa de evaporação Equação 1.b (g/s)

$\mathrm{k}^{\mathrm{ys}, \mathrm{Tgs}}$ Taxa de evaporação Equações 1.a e 1.c $(\mathrm{g} / \mathrm{s})$

$\mathrm{mg}_{\mathrm{g}} \quad$ Massa de gás $(\mathrm{g})$

$\mathrm{m}_{\mathrm{j}} \quad$ Massa do leito de jorro $(\mathrm{g})$

$\mathrm{m}_{\mathrm{s}} \quad$ Massa das partículas inertes $(\mathrm{g})$

qconv Calor perdido pelo leito de jorro (J/s)

Qsusp Vazão de suspensão ( $\mathrm{mL} / \mathrm{min})$

$\mathrm{t}$ tempo (s)

$\mathrm{Tg}_{\mathrm{e}} \quad$ Temperatura do gás na entrada $\left({ }^{\circ} \mathrm{C}\right)$

$\mathrm{Tg}_{\mathrm{s}} \quad$ Temperatura do gás na saída $\left({ }^{\circ} \mathrm{C}\right)$

$\mathrm{t}_{\mathrm{p}} \quad$ tempo a perturbação da pasta (s)

u Velocidade do gás na entrada $(\mathrm{m} / \mathrm{s})$

$\mathrm{UA}_{\mathrm{s}} \quad$ Umidade absoluta saída $\left(\mathrm{kg} \mathrm{H}_{2} \mathrm{O} / \mathrm{kg}\right.$ a.s)

$\mathrm{u}_{\mathrm{mj}} \quad$ Velocidade mínima de jorro $(\mathrm{m} / \mathrm{s})$

$\mathrm{UR}_{\mathrm{S}} \quad$ Umidade relativa saída $(\%)$

$\mathrm{Wg}_{\mathrm{e}} \quad$ Vazão mássica do gás na entrada do leito $(\mathrm{g} / \mathrm{s})$

$\mathrm{Wg}_{\mathrm{s}} \quad$ Vazão mássica do gás na saída do leito $(\mathrm{g} / \mathrm{s})$

Xbu Conteúdo de umidade do pó (\%) 
$\mathrm{x}_{\mathrm{e}} \quad$ Fração mássica de água líquida na entrada do leito de jorro

$\mathrm{x}_{\mathrm{s}} \quad$ Fração mássica de água líquida na saída do leito de jorro

$\mathrm{y}_{\mathrm{s}} \quad$ Fração mássica de água na fase vapor

$\rho_{p} \quad$ Densidade real das partículas $\left(\mathrm{kg} / \mathrm{m}^{3}\right)$

$\Delta \mathrm{P}_{\mathrm{L}} \quad$ Queda de pressão do leito $(\mathrm{Pa})$

$\Delta \mathrm{P}_{\max } \quad$ Queda de pressão máxima $(\mathrm{Pa})$

$\Delta \mathrm{P}_{\mathrm{mj}} \quad$ Queda de pressão mínimo jorro $(\mathrm{Pa})$

\section{REFERÊNCIAS}

ALMEIDA, A. R. F. Análise da Secagem de Pastas em Leito de Jorro. 2009. 165p. Tese (Doutorado em Engenharia Química) Universidade Federal de São Carlos, São Carlos, 2009.

DANTAS, T. N. P. Avaliação de Dados de Secagem de Suspensões de Polpas de Frutas em Leito de Jorro com Alimentação Intermitente. 2013. 83p. Dissertação (Mestrado em Engenharia Química) Universidade Federal do Rio Grande do Norte, Natal. 2013

FERNANDES, C. F. Análise da eficiência energética da secagem de pastas em leito de jorro. 2005. 96p. Dissertação (Mestrado em Engenharia Química), Universidade Federal de São Carlos, São Carlos. 2005.

FILHO, F. C. M. Avaliação da Aplicação de Modelos para a Secagem de Pastas e Suspensões em Leito de Jorro com Alimentação Contínua. 2013. 95p. Dissertação (Mestrado em Engenharia Química) - Universidade Federal do Rio Grande do Norte, Natal. 2013

FREIRE, J. T.; ALMEIDA, A. R. F.; FREIRE, F. B. Análise do regime transiente na secagem de pastas em leito de jorro com partículas inertes através do modelo CST. In: FREIRE, J. T et al. Aplicações em Sistemas
Particulados. São Carlos: Suprema, 2009. Vol. 1, Cap. 3, p. 74-112.

IMSL Library, MATH/LIB, Houston; 1991.

MATHUR, K. B.; EPSTEIN, N. Spouted Beds. New York. Academic Press, 1974.304 p.

MEDEIROS, M. F. D. Influência da Composição Química dos Materiais no Desempenho do Processo de Secagem de Polpas de Frutas em Leito de Jorro. 2001. 247p. Tese (Doutorado em Engenharia Química) - Universidade Estadual de Campinas, Campinas, 2001.

VIEIRA, G. N. A. Monitoramento em tempo real e simulação de controle da umidade de pós produzidos pela secagem de pastas em leito de jorro. 2015. 111p. Tese (Doutorado em Engenharia Química) - Universidade Federal de São Carlos, São Carlos. 2015.

\section{AGRADECIMENTOS}

Os autores deste trabalho agradecem a CAPES pelo apoio financeiro $\mathrm{e}$ ao DEQ/UFSCar. 